\title{
Peculiarities of spin reorientation in a thin YIG film
}

\author{
Ya.B. Bazaliy ${ }^{\mathrm{a}, \mathrm{b}}$, L.T. Tsymbal ${ }^{\mathrm{a}, \mathrm{c}, 1}$, A.I. Linnik ${ }^{\mathrm{a}}$ N.K. Dan'shin ${ }^{\text {a }}$ A.I. Izotov ${ }^{\mathrm{a}}$ \\ P.E. Wigen ${ }^{\mathrm{c}}$ \\ ${ }^{a}$ O. Galkin Donetsk Physics \& Technology Institute Nat.Ac.Sci. Ukraine, R.Luxemburg 72, Donetsk, 83114, Ukraine \\ ${ }^{\mathrm{b}}$ MSD, Argonne National Laboratory, 9700 S. Cass Ave. Argonne, IL 60439, USA \\ ${ }^{\mathrm{c}}$ Department of Physics, Ohio State University, 174 W. 18th Ave., Columbus, OH 43210, USA
}

\begin{abstract}
The issue of magnetic orientation transitions in thin films combines interesting physics and importance for applications. We study the magnetic transition and phase diagram of a $0.1 \mu \mathrm{m}$ thick (YLaGd) $)_{3}(\mathrm{FeGa})_{5} \mathrm{O}_{12}$ films grown on GGG substrate by liquid phase epitaxy. Observed transitions are compared with those in BiGa:TmIG thin films, studied in previous work by one of the authors. A general picture of orientation transitions in thin films of substituted YIG is discussed.
\end{abstract}

Key words: orientation phase transitions; thin magnetic films; garnets;

1. Orientational phase transitions (OPT) are an attractive experimental object because in their vicinity the magnetic anisotropy energy goes to zero, thus enabling an investigation of the smaller interactions in the system, which otherwise are masked in magnetic crystals $[1,2]$.

In this paper the OPTs in thin iron garnet films are investigated. Their application in industry propels interest in the physical processes inherent to such films. While spin reorientations are common in the orthoferrites, they are rare in bulk rare-earth iron garnets. However that does not mean that OPTs are irrelevant in the garnet films, because in the film geometry the dipole-dipole interactions start to play a defining role.

2. Single crystal magnetic films of cubic structure with the film normal $n$ parallel to the $\langle 111\rangle$ axis of the material are studied. Magnetocrystalline anisotropy in the film is a sum of a cubic-symmetry term that favors magnetization $M$ pointing in one of the eight $\langle 111\rangle$ directions and the uniaxial growth anisotropy en-

\footnotetext{
1 Corresponding author. Present address: O.Galkin Donetsk Physics\&Technology Institute of National Academy of Science of Ukraine, R.Luxemburg 72, Donetsk, 83114, Ukraine; E-mail: tsymbal@sova.fti.ac.donetsk.ua
}

ergy that favors $M\|n\|\langle 111\rangle$. The energy of the film can be written as a sum of cubic and uniaxial growth anisotropy energies, and dipole-dipole interaction energy or "shape anisotropy" energy (details in [3]):

$E=E_{c}+E_{u}+E_{d d}$

The last term leads to versatile domain structures which depend on the film thickness $d$, domain wall width $\delta$, and the ratio of the demagnetization energy constant $K_{d}=2 \pi M_{s}^{2}$ to the constants $K_{c}$ and $K_{u}$ of cubic and uniaxial magnetocrystalline anisotropy energies.

The first observation of the spontaneous OPTs in the garnet films was made in $[3,4]$. It was discovered that magnetization of the single crystal $\mathrm{Tm}_{2.14} \mathrm{Bi}_{0.80} \mathrm{Fe}_{3.1} \mathrm{Ga}_{1.9} \mathrm{O}_{12}$ films rotates from in-plane to the normal direction through two second-order transitions at $T_{1}=100 \mathrm{~K}$ and $T_{2}=125 \mathrm{~K}$. External magnetic field $H \|\langle 111\rangle$ decreases the transition temperatures.

In contrast, a pure YIG films exhibit no OPT in the temperature interval $4 \mathrm{~K}-300 \mathrm{~K}$. A question arises, whether there is any fundamental difference between the physics of differently substituted YIG films? Ana- 
lyzing Eqn.(1)the only term favoring $M$ being aligned parallel to the film plane is the dipole-dipole interaction. It is known that for the simpler uniaxial case $\left(K_{c}=0\right)$ the magnetization is indeed lying in the plane if two conditions are met [5]:

$Q=\frac{K_{u}}{2 \pi M^{2}}<1, \quad d<d_{*} \approx \delta$

The quality factor $Q$ and critical thickness $d_{*}$ are functions of temperature and if e.g. $Q(T)=1$ at some temperature, a phase transition with rotation of $M$ out of plane will occur. The cubic anisotropy term should complicate the analysis and may lead to the appearance of several transitions in correspondence with the experiments $[3,4]$.

A possible way to test this scenario is to decrease the value of $Q$ or film thickness $d$ and observe an increasing of transition temperatures. In accord with this idea a very thin $(\mathrm{YLaGd})_{3}(\mathrm{FeGa})_{5} \mathrm{O}_{12}$ film grown by liquid phase epitaxy on a [111] oriented gadoliniumgallium garnet (GGG) single crystal substrate was studied. This composition was chosen to make the uniaxial anisotropy constant $K_{u}$ as small as possible. The thickness of the film was $d \approx 0.1 \mu \mathrm{m}$, the sample was a disk of $5 \mathrm{~mm}$ in diameter. The conditions required by Eqn.(2) were satisfied for this film with $Q<<1$

We measured magnetic susceptibility $\chi(H)$ at the $5 \mathrm{MHz}$ and the soft FMR mode frequency $\Omega(H)$ (Fig 1 ). In the temperature range from $77 \mathrm{~K}$ to $450 \mathrm{~K}$ no spontaneous OPTs were observed in accord with what was stated above. To move towards our goal the sample was put into a magnetic field $H\|n\|\langle 111\rangle$ that promotes orientation transition between in-plane and out of plane phases. In our experiments the direction of $H$ and $\langle 111\rangle$ coincided within a $3^{\prime}$ accuracy. Experimental $\chi(H)$ dependencies are shown on Fig. 1. The sharp dips mark

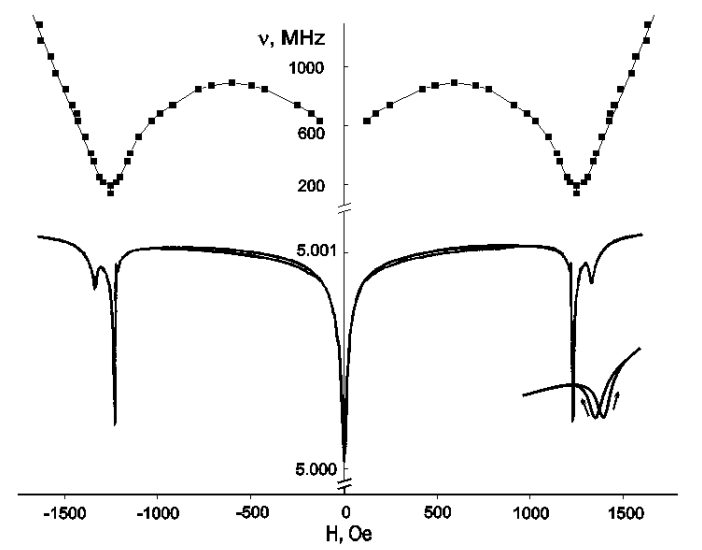

Fig. 1. Magnetic field dependence of the FMR frequency $\Omega(H)$ (upper curve) and $\gamma(H)=1 / \sqrt{1+4 \pi \chi(H)}$ (middle curve) at $T=293 K$. Lower curve: $\gamma(H, T=125 K)$ near $H_{*}$.

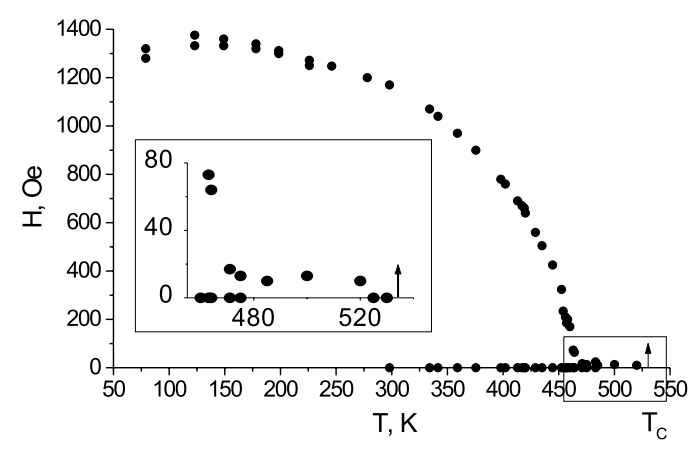

Fig. 2. Phase diagram of a thin YIG film in $\mathbf{H} \|\langle 111\rangle$. Only positive values of field are shown. Inset shows how phase boundary curves near $T_{*} \approx 465 \mathrm{~K} . T_{c}$ is the Curie temperature.

the phase transitions at $H= \pm H_{*}$ and $H=0$ (see [6]).

The transition at $H_{*}$ is identified as the field for which the sample enters into the monodomain state with $M \|\langle 111\rangle$. This transition is observed as a minimum on the $\Omega(H)$ curve (Fig. 1). The absence of hysteresis confirms the second-order nature of the transition. The transition at $H=0$ is seen very clearly and happens without hysteresis. Experimentally it must be identified as a second-order transition.

After performing the measurements of $\chi(H, T)$ at many temperatures an $(H-T)$ phase diagram of the film can be constructed (Fig. 2). The $H_{*}(T)$ line separates the monodomain $M \|\langle 111\rangle$ state for $H>H_{*}$ from the multi-domain state with tilted $M$ for $H<H_{*}$.

3. The $H_{*}(T)$ curve has a sharp turn near $T_{*} \approx$ 465K (see inset in Fig.2). It's overall behavior for $T<$ $T_{*}$ is similar that observed in $[3,4]$. The large values of $T_{1}$ would be in accord with the arguments about the consequences of lowering $K_{u}$ and $d$. Application of the magnetic field merely enables one to reveal those large $T_{1}$ while not going above room temperature. However this analogy is far from complete. In particular the sharp turn of $H_{*}(T)$ for $T_{*}$ approaching $T_{c}$ (see inset)and it's subsequent behavior has no analog in previous films $[3,4]$. The differences may be the consequences of $T_{1,2}$ being close to the Curie temperature $T_{c}$ of the material where changes in the magnitude of $M$ must be accounted for. Full understanding of the $H_{*}(T)$ behavior in YIG films calls for more investigations.

Y.B. was supported by the U.S. Dept. of Energy, Office of Science, under Contract No. W-31-109-ENG38.

\section{References}

[1] V.Buchelnikov et al., Physiks - Uspekhi, 39 (1996) 547

[2] V.Buchelnikov et al., Physiks - Uspekhi, 42 (1999) 957

[3] R.Bornfreund et al., JMMM 151 (1995) 181 
[4] R.Bornfreund et al., IEEE 28 (1992) 2991

[5] A. Hubert, R. Schafer "Magnetic Domains", Springer (1998)

[6] V.Buchelnikov et al., JETP (2002), to be published 